\title{
The Dependence of Confinement Energy on the Size of Quantum Dots
}

\author{
H. I. Ikeri ${ }^{1 *}$, A. I. Onyia ${ }^{2}$, and O. J. Vwavware ${ }^{3}$ \\ ${ }^{1,2,3}$ Department of Industrial Physics, Enugu State University of Science and Technology, Enugu, Nigeria \\ *Corresponding author: ifeanyihenry75@yahoo.com
}

Available online at: www.isroset.org

Received: 12/Apr/2019, Accepted: 21/Apr/2019, Online: 30/Apr/2019

\begin{abstract}
Theoretical study of the dependence of confinement energy on the size of Quantum dots (QDs) using quantum mechanical approach is presented. A simple model obtained for confinement energy is generally found to be in good agreement with the predicted inverse quadratic dependence on the dot size. Thus optical and electronic behavior of QDs can be engineered during manufacturing to meet specific applications. It is found that energy levels of the charge carriers within QDs are increased yielding to discrete energy states for electrons and holes. Therefore, QDs can emit and absorb light at specific wavelengths which are related to QD size. The plots for ground state confinement energy as a function of dots radius for CdSe, GaAs and ZnS QDs show monotonous decay curves. Thus as the dot radius increases, the ground state confinement energy decreases exponentially but never reaches zero. Thus, charge carriers in quantum dots possess non-zero minimum energy state in consistence with the infinite potential well. It can be observed that making the dot size large enough the effect of size on confinement energy is very small for different QDs. This is because as QDs grow larger their energy levels move closer and form a near continuum. In addition, among the QDs considered the degree of confinement on the CdSe and GaAs QDs strongly indicates that their optical wavelength can be extended to match the solar spectrum for multi-junction solar cells applications. This improves the solar to electricity conversion efficiency by harvesting multiple portions of solar spectrum.
\end{abstract}

Keywords: Nanotechnology, Quantum Dot, Confinement, Confinement Energy, Potential well, Solar cell, and Quantum Mechanics.

\section{INTRODUCTION}

In this era of miniaturization and nanotechnology referred to as Nano-age, quantum dots unique electronic and optical properties and potential applications in modern technology have attracted significant interest both for fundamental research and Nano electronic devices and have revolutionized a new research in condensed matter physics.

Quantum dots are fundamentally interesting, owing to their excellent electronic and optical properties [1], which intermediate between those of bulk materials and discrete molecules that are closely related to the dot size [2]. This allows properties such as emission and absorption spectra to be highly tunable [3]. The discrete quantized energy levels of quantum dots relate them closely to atoms than their bulk semiconductors [4]. Owing to this resemblance between the QDs and the atoms they are often referred to as artificial atoms [5], and thus enables the study of electronic systems without the presence of nucleus that affects the electrons [6] .The band gap which determines the conductive behavior of QDs depends primarily on the confinement energy of the charge carriers (electrons and the hole) [7]. For this reason, a deeper understanding of confinement energy of QDs is crucial.
Quantum dots are semiconductor Nano particles that exhibit three-dimensional confinement [8], and thus represent the ultimate limit of low dimension structures due to their three dimensional carrier confinement [9]. The charge carriers become spatially confined when the diameter of the bulk semiconductor approaches the de Broglie wavelength of electrons in the conduction band or when the bulk semiconductor becomes smaller than the exciton Bohr radius (which is an average distance between an electron in the conduction band and the hole it leaves behind in the valence band) [10]. The confinement of charge carriers in quantum dots leads to size quantization [11], and this have important repercussions on the absorption and emission spectra which are now blue shifted with decreasing dot size [12] as shown in Fig 1.This means the biggest quantum dots produce red light while the smallest dots make blue light. Thus band gap is tunable by changing the size of quantum dots (QDs) based on the quantum confinement effect which plays a fundamental role in optical and electrical properties of QDs. 


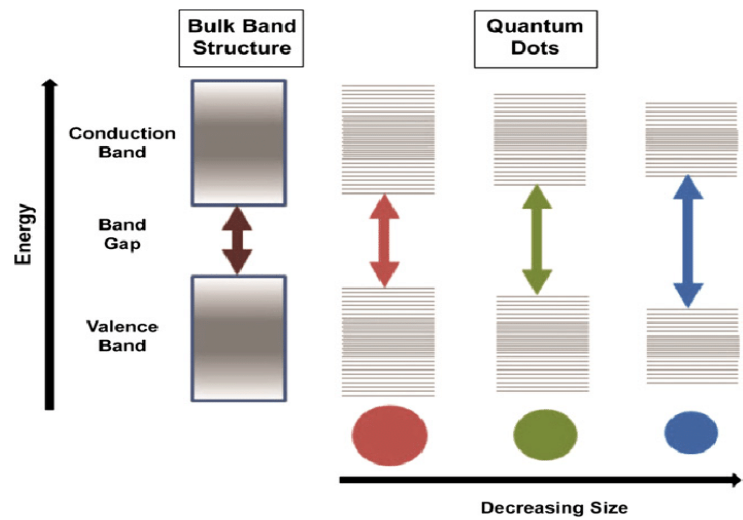

Figure 1: Optical Spectra of Quantum Dots with Decreasing Size.

The ability to tune the optical bandgap of QDs over a wide range of energy levels particularly makes QD a promising material for high efficient solar cell as their energy spectrum can be tuned to match spectral distribution of solar spectrum. Such QD-based solar cells that utilize quantization effect to enhance solar to electricity energy conversion efficiencies form the basis for third generation solar cells. [13].

The name quantum dot demonstrates some of the properties of the system. The word "dot" refers to the system spatial structure [14], that is more like a single point (zero dimensional), which implies confinement in all three spatial dimensions while the word quantum indicates that the actual size of the system is very small [15], and reviews the physics that governs the system behavior otherwise known as quantum mechanics. This makes QDs an excellent structure for studying real-life quantum effects and perfect examples of quantum mechanical systems. Thus, quantum mechanical theory should be adequately utilized to describe the behavior of such a quantum structures.

This article provides a comprehensive study of dependence of confinement energy on quantum dot size using quantum mechanical approach.We organized the work in sections as follows. The introduction and related literature are discussed in section I.Section II provides detailed theoretical framework which described the system behavior followed by materials and methods described in section III which deals with the computation details and method of calculation. A detailed computational results as obtained for the considered QDs has been provided and been thoroughly discussed in section IV. In the concluding section $\mathrm{V}$ the observed results are summarized.

\section{II.THEORETICAL FRAMEWORK}

Quantum dots confine their charge carriers in a small spatial domain known as quantum box. Because of this small length scale, QDs exhibit quantum mechanical properties and the charge carriers can be described qualitatively as particles in an infinite potential well. In order to obtain the confinement energy of these charge carriers within the dot, we solve the Schrodinger quantum mechanics in a spherical infinite potential well.

Quantum dots are two particle systems consisting of electron and hole. The two particles interact through the potential given by Coulombs law:

$$
V(r)=\frac{e^{2}}{k r}(1)
$$

where

- $\quad \mathrm{e}$ is the charge of electron

- $\mathrm{r}$ is the distance between the electron and the hole, and

- $\mathrm{k}$ is the dielectric constant of semiconductor

The electron and the holehave been approximated as a single quasi-particle bounded by Coulomb force moving freely in a spherical box. This particle is in a periodic potential caused by the semiconductor crystal and thus have reduced effective mass given by

$$
\mu=\frac{m_{e}^{*} m_{h}^{*}}{m_{e}^{*}+m_{h}^{*}}(2)
$$

In general, the dynamics of a particle in a spherical potential are governed by a Hamiltonian of the form:

$$
\frac{\hbar^{2}}{2 m} \frac{d^{2}}{d x^{2}}+v(x)=H(3)
$$

The confinement energy (energy state) of the charge carriers (electron and hole) are found by solving Schrodinger equation with this Hamiltonian given as:

$$
\frac{\hbar^{2}}{2 m} \frac{d^{2} \varphi(x)}{d x^{2}}+v(x) \varphi(x)=E \varphi(x)(4)
$$

We rearrange the equation to have:

$\frac{\hbar^{2}}{2 m} \frac{d^{2} \varphi(x)}{d x^{2}}+[E-v(x)] \varphi(x)=0(5)$

Due to the space confinement of the charge carriers within the QD the boundary conditions of confinement apply. The confined particle is within the regions $x=0$ and $x=L$ with potential energy $v=0$ bounded by infinite high potential barriers, that is, $V=\infty$ for $x \leq 0$ and $V=$ $\infty$ for $x \geq L$. In the regions for which the potential is infinite, the wave function $\varphi(x)$ will be zero. The boundary conditions are thus wave function at $x=0$ and the wave function at $x=l$ is zero.In the region $0<x<L$, the potential function varnishes i.e $v=0$ so the Schrodinger equation inside the box becomes

$$
\frac{\hbar^{2}}{2 m} \frac{d^{2} \varphi(x)}{d x^{2}}+E \varphi(x)=0(6)
$$


Usually, in QD there is spherical confinement and not rectangular. We therefore model QD as a spherical three dimensional infinite potential well.The Schrodinger equation was then solved for its energy states. The quantized energy state solution of a spherical QD for the electron and hole is

$$
E_{n, m}=\frac{\beta_{n, m \hbar^{2}}^{2}}{2 \mu R^{2}}(7)
$$

where

- $\quad \beta_{n, m}^{2}$ is the mth root of the spherical Bessel function of quantum number $\mathrm{n}$

- $R$ is the radius of the QD

- $\mu$ is the reduced effective mass

- $E_{n, m}$ is the confinement energy (energy state) of the electron and hole.

The ground state confinement energy which is the commonly observed state (corresponding to $\beta_{1,0}^{2}=\pi$ ) can easily be found, taking $\mathrm{n}=1$ and is given as

$$
E_{1,0}=\frac{\pi \hbar^{2}}{2 \mu R^{2}}(8)
$$

\section{MATERIALS AND METHODS}

The material parameters we have used for the study of dependence of confinement energy on QD size are: effective mass of electron, $m_{e}^{*}$, effective mass of hole, $m_{h}^{*}$, rest mass of electron, $\mathrm{m}_{\mathrm{o}}$, for $\mathrm{CdSe}, \mathrm{ZnS}$ and GaAs quantum dots as shown in the table 1.We have approximated QD as a spherical infinite potential well and then solved the time independent Schrodinger equation. A simple model for the confinement energy of the electrons and holes within the QD was obtained.

Variations in ground state confinement energy as a function of dot sizes were also calculated for the three considered QDs using equation 8. In arriving at the results, several parameters were used, for $\mathrm{CdSe}, \mathrm{GaAsand} \mathrm{ZnS}$ as shown in the tables below.

Table 1. The Material Parameters used for the Study.

\begin{tabular}{lcc}
\hline QDs & $m_{e}^{*}$ & $m_{h}^{*}$ \\
\hline $\mathrm{CdSe}$ & $0.13 \mathrm{~m}_{\mathrm{o}}$ & $0.45 \mathrm{~m}_{\mathrm{o}}$ \\
$\mathrm{ZnS}$ & $0.34 \mathrm{~m}_{\mathrm{o}}$ & $0.23 \mathrm{~m}_{\mathrm{o}}$ \\
$\mathrm{GaAs}$ & $0.06 \mathrm{~m}_{\mathrm{o}}$ & $0.51 \mathrm{~m}_{\mathrm{o}}$ \\
\hline
\end{tabular}

\section{RESULTS AND DISCUSSION}

A simple model obtained for confinement energy is generally found to be in good agreement with the predicted inverse quadratic dependence on the dot size. Thus as the dot size $\mathrm{R}$ is reduced, the confinement energy becomes larger by the factor $1 / \mathrm{R}^{2}$. It follows that conductivity of $\mathrm{QD}$ which depends primarily on the confinement energy also exhibits size dependentbehavior. As a result of this phenomenon the electronic and optical properties of QDs can be engineered to meet specific applications, thus making QDs suitable materials for nano-electronic devices. It is found that energy levels of the charge carriers within QDs are increased yielding to discrete energy states for electrons and holes. Thus,QDs can emit and absorb light at specific wavelengths. The dependence of confinement energy on the size of QD is shown on the graph of ground state confinement energy as a function of the radius plotted for $\mathrm{CdSe}$, GaAs and $\mathrm{ZnS}$ QDs in Fig 2. The results show exponential decay curves. As the dot radius increases, the ground state confinement energy decreases exponentially but never reaches zero. Thus, the charge carriers in quantum QDs possess a non-zero minimum energy in consistence with the particle in a potential well.

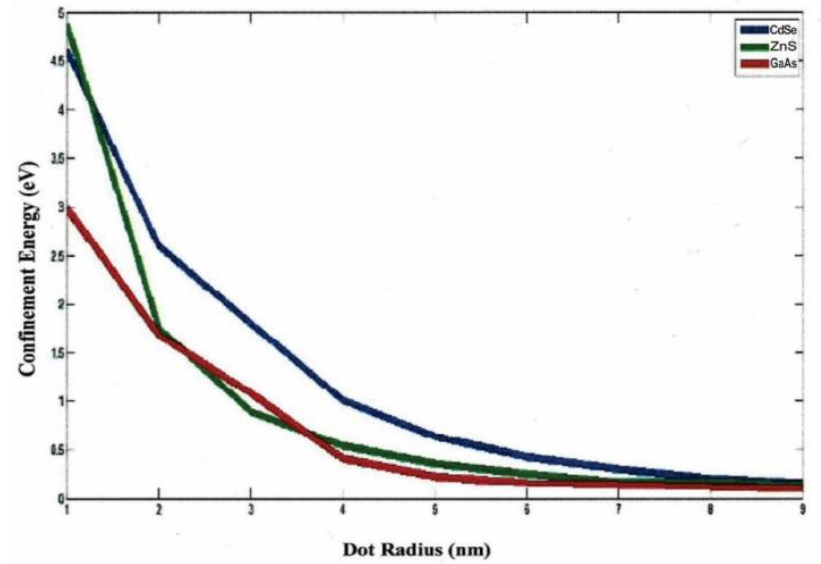

Figure 2: Confinement energy as a function of the dot radius for $\mathrm{CdSe}, \mathrm{ZnS}$ and GaAs Quantum Dots.

It is clear fromFig 2,that as the size of quantum dots is increased a sudden decrease in confinement occurs and then it becomes constant for large value of QDs size and the quantization effect on the confinement energy becomes smaller. This is because as QDs grow larger their energy levels move closer together and form a near continuum indicating a weak confinement regime. In addition, among the QDs considered the degree of confinement on the CdSe and GaAs QDs indicates that they possess optical trait for possible extension of their wavelength to match the spectral distribution of solar spectrum. This is particularly beneficial for third generation solar cells in which the solar to electricity energy conversion efficiencies will be significantly improved over the theoretical efficiency limit of $31 \%$ for conventional solar cells.

\section{V.CONCLUSION}

Confinement energy can be seen as a consequence of spherical infinite potential well model. A simple model obtained is in good agreement with the experimental results. 
Thus, confinement of charge carriers in semiconductor quantum dots increases dramatically with decrease in its size (radius) and shows exponential dependence on dot radius.It is found that energy levels of the charge carriers within QDs are increased yielding to discrete energy states for electrons and holes. Thus, QDs can emit and absorb light at specific wavelengths. A close look at the computed ground state confinement energy as a function of dot size shown in Fig. 2 indicates that charge carrier in QD possess a non-zero minimum energy state. In addition, the degree of confinement on CdSe and GaAs QDs implies that their optical bandgap can be extended to match the spectral distribution of solar spectrum for mult-junction solar cells application.

\section{REFERENCES}

[1.] K. E. Aderson, W.E. Picket, "Quantum Confinement in CdSeNanocrystallites",Journal of Nano-Crystalline Solids, pp. 299-302, 1105-1110 2002.

[2.] J Sinclair, D. Dagotto, "An Introduction to Quantum Dots: Confinement, Synthesis, Artificial Atoms and Applications", Solid State II Lecture Notes, University of Tennessee, Knoxville, 2009.

[3.] K., Grieve, P., Mulvaney, F., Grieser, "Synthesis and Electronic Properties of Semiconductor and Interface Science",pp.168- 172, 2000.

[4.] E. O., Chukwuocha S. Harry, "Theoretical Studies on the Effect of Confinement on the Wavelength of CdSe Quantum Dots. Int. Journal of Science and Technology Research, vol. 1 No. 7, pp.21-24. 20

[5.] .H, Davies, "The Physics of Low Dimensional Semiconductors;" An introduction $\left(6^{\text {th }}\right.$ reprint ed, ) Cambridge University Press. 2006.

[6.] C. Orion, "Understanding Electronic Systems in Semiconductor Quantum Dots," PhysicaSripta, Vol. 88, No.5, 2013.

[7.] L. Brus, "Electron-Electron and Electron-Hole Interactions in small crystallites: the size Dependent of the Lowest Excited Electronic State", Journal of Chemical Physics, vol. 80, No. 9, pp. 4403, 1984.

[8.] N.Pandey, A.Dwivedi, "Theoretical study of Dependence of Wavelength on Size of Quantum Dot,"International Journal of Scientific Research and Development vol. 4, issue 01, 2016.

[9.] M. Wise, "Calculation of Electronics Structure of a Spherical Quantum Dots." InternationalJournal of Condensed Matter Physics, vol. 3, pp.556-560, 2000.

[10.] H.I., Ikeri, A.I.,Onyia, "Theoretical Investigation of Size Effect on Energy Gap of Quantum Dots Using Particle in a Box Model", Chalcogenide Letters, Vol. 14, No.2, pp. 49 - 54, 2017.

[11.] A.I. Onyia, H. I. Ikeri, "Theoretical Study of Quantum Confinement Effect on Quantum Dots Using Particle in a Box Model," Journal of Ovonic Research, vol. 14, No.1, pp. 49 54, 2018.

[12.] A. Alivisation, "Semiconductor Cluster, Nanocrystals and Quantum Dots." Journal of Applied Physics vol. 8, pp.127 134, 1996

[13.] A. J. Nozik, "Quantum Dot Solar Cells," Physica E, vol. 14, pp.115-120, 2002.

[14.] E.O Chukwuocha, M.C. Onyeaju, "Simulation of Quantum Dots in a Confinement Regime", Int. Journal of Applied Science and Engineering Research, vol. 1 No.6, pp.791. 2012.
[15.] B. Billaud, T. Troung, "Some Theoretical Result in Semiconductor Spherical Quantum Dots."Computational Material Science, vol. 49, pp.332-335, 2010.

\section{AUTHORS PROFILE}

Ikeri Henry is presently pursuing Ph.D degree in Solid State Physics from Enugu State University of Science and Technology, Enugu, Nigeria. His research work focuses on the impact of Quantum Dots on Efficiency Optimization for third Generation Solar Cells. He obtained his M.Sc. Solid State Physics with distinction in 2017 from Enugu State University of Science and Technology, Enugu, Nigeria. His areas of research interest are Nanotechnology, Material Science, Nano Electronics, and Solar Technology. He has published a number of research papers in a reputed peer review academic national and international Journals/Conferences. He is working as a Lecturer and currently Head of Department of Electrical/ElectronicsEngineering,Eastern Polytechnic Port Harcourt, Rivers State, Nigeria.

DR. Onyia Augustine did M.Sc. in Physics from Department of Physics and Astronomy, University of Nigeria Nssuka with specialization in Solid State Physics. He completed his Ph.D Solid State Physics in 2016 from Ebonyi State University of Science and TechnologyEbonyi, Nigeria. His areas of research interest are Thin FilmFabrication, Nanotechnology, Material Science and Solar Technology. He has published many research papers in reputed, peerreviewed and citation index local and international journals/conferences. Dr. Onyia is the Head of Department of Industrial Physics Enugu State University of Science andTechnology. 